\title{
Instanton effects on the twist-three light-cone distribution amplitudes of pion and light scalar mesons above $1 \mathrm{GeV}$
}

\author{
Jin Zhang ${ }^{1}$, Zhu-Feng Zhang ${ }^{2}$ and Hong-Ying Jin ${ }^{1}$, \\ ${ }^{1}$ Institute of Modern Physics, Zhejiang University, Hangzhou, Zhejiang, China \\ ${ }^{2}$ Department of Physics, Ningbo University, Ningbo, Zhejiang, China
}

\begin{abstract}
By use of single instanton approximation the twist-3 light-cone distribution amplitudes of pion as well as the p-wave mesons, i.e., $f_{0}(1370), K_{0}^{*}(1430)$ and $a_{0}(1450)$ are investigated within the framework of QCD moment sum rules with inclusion of instanton effects based on the valence quark model. Results show that there is much change on light-cone distribution amplitudes by the isospinand chirality-dependent instanton contribution compared with the instanton-free ones. We find the intanton involved twist-3 LCDAs are non-positive-definite within some range of momentum fraction and there are rapid changes at two ends of momentum fraction. To guarantee the convergence of moments by the method in this work a low instanton density should be adopted, for instance $n_{c}=$ $\frac{1}{2} \mathrm{fm}^{-4}$ is welcomed. Possible ingredients which might have impact on the results are briefly discussed. These light-cone distribution amplitudes may be helpful to analyze exclusive heavy flavored processes.
\end{abstract}

PACS numbers: 


\section{INTRODUCTION}

The hadronic light-cone distribution amplitude(LCDA or light-cone wave functions) which parameterize the non-perturbative effects play an important role in understanding exclusive hard processes in QCD [1 3]. The LCDA is also one of the key ingredients in QCD factorization approach[4] for describing exclusive hadronic B decays which are helpful to ascertain the quark content of light scalars [5-7] and other observables of phenomenological interest. Therefore if there is more information on LCDA there will be more complete study on heavy-flavored exclusive processes consequently many important parameters in standard model. To extract LCDA the nonperturbative method should be employed due to its nonperturbative nature. The QCD sum rules[8] have been proved to be very successful one in obtaining useful information in hadronic region [9]. While it was found later after its born in order to produce reasonable results of lowest-lying states of pseudoscalar channel the instanton effect 10 14] should be taken into account in QCD sum rules[15] $]^{1}$. Then the role of instanton in QCD sum rules is extensively investigated[17, 18]. Recently it was noticed that instanton may be helpful in lifting the mass degeneration in light scalar mesons above $1 \mathrm{GeV}[19]$. The calculation of LCDAs within the framework of QCD sum rules was introduced in[20] by studying the correlation function of currents with derivatives in this way the desired Gegenbauer coefficients can be expressed in terms of the moments derived from QCD sum rules. Using this method Chernyak and Zhitnitsky $(\mathrm{CZ})$ were able to describe many experimental data available at that time[21]. Marrying with lattice simulation the first two Gegenbauer coefficients of pion and kaon were studied[22] under this method.

In analyzing $\mathrm{B}$ decays to p-wave mesons [23, 24] (more complete review on B decays, see[25]) such as $f_{0}(1370), K_{0}^{*}(1430), a_{0}(1450)$ the LCDAs are important input. Having noticed the significant implication of instanton in producing the realistic mass spectral of $f_{0}, K_{0}^{*}$ and $a_{0}$ above $1 \mathrm{GeV}[19]$, in this paper we will make some effort to investigate the instanton effects on the twist-three LCDAs of these mesons by the CZ method. The work in this paper can be

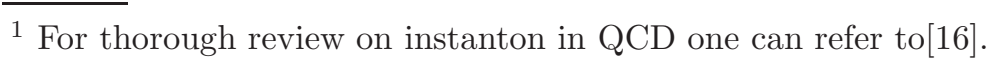


regarded as partly pursuing of Ref. [19] and the quark content are assigned as follows

$$
\begin{aligned}
f_{0} & =\frac{1}{\sqrt{2}}(u \bar{u}+d \bar{d}), \\
K_{0}^{*} & =d \bar{s} \\
a_{0} & =\frac{1}{\sqrt{2}}(u \bar{u}-d \bar{d}),
\end{aligned}
$$

The LCDAs of pion, as a widely used quantity in exclusive processes, naturally attach attentions based on various methods from instanton model[26]. To this point we would like to stress that instanton is crucial in reproducing reasonable results of pion and its partners in $0^{-}$channel by QCD sum rules[15]. Perturbatively the intuition is that due to the chirality suppress there should be larger size of $s \bar{s}$ component in $\omega$ than $\eta$. If it were the case $\omega$ would be close to $\phi$ but the fact is that $\omega$ nearly degenerates with $\rho$ while there is considerable mass gap between $\omega$ and $\phi$. The empirical spectrum is that $\eta$ is nearly pure $\mathrm{SU}(3)$ octet while much smaller $s \bar{s}$ component in $\omega$. Then there arise the puzzle why there is much larger splitting between pion and $\eta$ than that of $\rho$ and $\omega$ which is difficult to explain perturbatively. The puzzle can be solved if the instanton is considered. Due to the definite chirality of fermion zero mode there is direct instanton contribution to pseudoscalar channel but not in the vector and tensor ones and additionally this instanton-induced correction is isospin-dependent thus pion and $\eta$ are lighter than $\rho$ and $\omega$ and there is larger mass gap between pion and $\eta$ than $\rho$ and $\omega$. Hence pion provides a natural laboratory to study instanton and it is expected there should be some effects of instanton on its twist-3 LCDAs. Therefore it is meaningful to analyze the twist-3 pion LCDAs by CZ method with inclusion of instanton. In fact the isospin-dependence of instanton effects is also important to reproduce a realistic mass gap between $a_{0}$ and $f_{0}$ in $0^{+}$channel above $1 \mathrm{GeV}$ by QCD sum rules assuming small size $s \bar{s}$ mixing into $f_{0}[19]$. The quark content of pion is as usual

$$
\pi^{0}=\frac{1}{\sqrt{2}}(u \bar{u}-d \bar{d})
$$

It is hoped these twist-3 LCDAs may shed some light on the exclusive hadronic B decays. We would like to emphasize that in QCD language a real hadron should be described by a set of Fock states which each one has the same quantum number as the hadron. For example

$$
\left|K_{0}\right\rangle=\psi_{d \bar{s}}^{K}|d \bar{s}\rangle+\psi_{d \bar{s} g}^{K}|d \bar{s} g\rangle+\psi_{d \bar{s} q \bar{q}}^{K}|d \bar{s} q \bar{q}\rangle+\ldots
$$


It is no doubt there are also twist-3 LCDAs introduced by higher Fock states but we will not consider them here. In other words we deal with leading Fock states or the valence quarks of the hadrons. Additionally there were some efforts in obtaining pion LCDAs from holographic QCD methods [27] and nonlocal condensates based on QCD sum rules[28] as well as some work on the shape of pion LCDAs[29]. These work gives some insights of pion LCDAs to us.

The outline of this paper is as follows: in Sec. II we present the sum rules with inclusion of instanton contributions to obtain the LCDAs of $f_{0}, K_{0}^{*}, a_{0}$ and pion. In Sec. III the numerical results and discussions will be presented, finally we summarize our conclusions in Sec. IV. An appendix is given to show the vanishing of instanton contribution to tensor moment sum rules within the method.

\section{BASIC FORMULAS}

\section{A. moments and light-cone distribution amplitudes}

Firstly we define the decay constants of scalar meson $\mathrm{S}$ and the pseudoscalar meson $\mathrm{P}$

$$
\begin{aligned}
\left\langle 0\left|\bar{q}_{2} q_{1}\right| S(p)\right\rangle & =m_{S} f_{S}, \\
\left\langle 0\left|\bar{q}_{2} i \gamma_{5} q_{1}\right| P(p)\right\rangle & =\frac{f_{P} m_{P}^{2}}{m_{1}+m_{2}},
\end{aligned}
$$

where $m_{S}, m_{P} m_{1}$ and $m_{2}$ are the mass of scalar meson, pseudoscalar meson, $q_{1}$ and $q_{2}$, respectively. The twist-3 LCDAs $\phi_{S}^{s}(u)$ and $\phi_{S}^{\sigma}(u)$ for the scalar meson $S$ with quark content $q_{1} \bar{q}_{2}$ are defined as [6]

$$
\begin{aligned}
\left\langle 0\left|\bar{q}_{2}\left(z_{2}\right) q_{1}\left(z_{1}\right)\right| S(p)\right\rangle & =m_{S} f_{S} \int_{0}^{1} d u e^{i\left(u p \cdot z_{2}+\bar{u} p \cdot z_{1}\right)} \phi_{S}^{s}(u) \\
\left\langle 0\left|\bar{q}_{2}\left(z_{2}\right) \sigma_{\mu \nu} q_{1}\left(z_{1}\right)\right| S(p)\right\rangle & =-m_{S} f_{S}\left(p_{\mu} z_{\nu}-p_{\nu} z_{\mu}\right) \int_{0}^{1} d u e^{i\left(u p \cdot z_{2}+\bar{u} p \cdot z_{1}\right)} \frac{\phi_{S}^{\sigma}(u)}{6}
\end{aligned}
$$

the two twist-3 two-particle LCDAs $\phi_{P}^{p}(u)$ and $\phi_{P}^{\sigma}(u)$ of pseudoscalar meson are defined as [30]

$$
\begin{aligned}
\left\langle 0\left|\bar{q}_{2}\left(z_{2}\right) i \gamma_{5} q_{1}\left(z_{1}\right)\right| P(p)\right\rangle & =\frac{f_{P} m_{P}^{2}}{m_{1}+m_{2}} \int_{0}^{1} d u e^{i\left(u p \cdot z_{2}+\bar{u} p \cdot z_{1}\right)} \phi_{P}^{p}(u), \\
\left\langle 0\left|\bar{q}_{2}\left(z_{2}\right) \sigma_{\mu \nu} \gamma_{5} q_{1}\left(z_{1}\right)\right| P(p)\right\rangle & =-\frac{i}{3} \frac{f_{P} m_{P}^{2}}{m_{1}+m_{2}}\left(p_{\mu} z_{\nu}-p_{\nu} z_{\mu}\right) \int_{0}^{1} d u e^{i\left(u p \cdot z_{2}+\bar{u} p \cdot z_{1}\right)} \phi_{P}^{\sigma}(u),
\end{aligned}
$$


where $u$ always refers to the momentum fraction carried by one quark and $\bar{u}=1-u$ is another quark momentum fraction; $z=z_{2}-z_{1}$. Noticing the gauge-invariant Wilson path-ordered integral

$$
\left[z_{2}, z_{1}\right]=P \exp \left[i g \int_{z_{1}}^{z_{2}} d \sigma_{\mu} A^{\mu}(\sigma)\right] .
$$

has been suppressed. The normalization of these four twist-3 LCDAs are

$$
\begin{aligned}
& \int_{0}^{1} d u \phi_{S}^{s}(u)=\int_{0}^{1} d u \phi_{S}^{\sigma}(u)=1 \\
& \int_{0}^{1} d u \phi_{P}^{p}(u)=\int_{0}^{1} d u \phi_{P}^{\sigma}(u)=1
\end{aligned}
$$

To proceed firstly we remind the reader that there is no pure zero-mode contribution to tensor moment sum rules(see the appendix) since instanton effect is chirality-dependent thus we do not consider $\phi_{S}^{\sigma}(u)$ and $\phi_{P}^{\sigma}(u)$ here. For the instanton-free tensor moment sum rules to calculate twist-3 LCDAs of p-wave mesons above $1 \mathrm{GeV}$ one can refer to Ref.[31]. For simplicity in this paper we study the two $\phi_{S}^{s}(u)$ and $\phi_{S}^{p}(u)$. In fact we can only concentrate on the scalar moment sum rules because the pseudoscalar one can be deduced from the scalar one by some appropriate substitutions, therefore in following we deal with the scalar sum rules only.

Generally the twist-three $\operatorname{LCDA} \phi_{S}^{s}(u)$ has the following form

$$
\phi_{S}^{s}(u, \mu)=1+\sum_{n=1}^{\infty} a_{n}(\mu) C_{n}^{1 / 2}(2 u-1),
$$

where $C_{n}^{1 / 2}(x)$ is Gegenbauer polynomials of order $1 / 2$, the lowest ones are 32 ]

$$
\begin{gathered}
C_{0}^{1 / 2}(x)=1, \quad C_{1}^{1 / 2}(x)=x, \quad C_{2}^{1 / 2}(x)=\frac{1}{2}\left(3 x^{2}-1\right) \\
C_{3}^{1 / 2}(x)=\frac{5}{2} x^{3}-\frac{3}{2} x, \quad C_{4}^{1 / 2}(x)=\frac{1}{8}\left(35 x^{4}-30 x^{2}+3\right)
\end{gathered}
$$

and the orthogonality relation is

$$
\int_{-1}^{1} C_{n}^{1 / 2}(x) C_{m}^{1 / 2}(x) d x=\frac{2}{2 n+1} \delta_{n m} .
$$

From Eq.(3) one can easily derive

$$
\left\langle 0\left|\bar{q}_{1}(0)(i z \cdot \overleftrightarrow{D})^{n} q_{2}(0)\right| S(p)\right\rangle=m_{S} f_{S}(p \cdot z)^{n}\left\langle\xi_{s}^{n}\right\rangle
$$


where

$$
\begin{array}{r}
\overleftrightarrow{D}=\vec{D}_{\mu}-\overleftarrow{D}_{\mu}, \quad \vec{D}_{\mu}=\vec{\partial}_{\mu}-i g A_{\mu}^{a} t^{a} \\
\left\langle\xi_{s}^{n}\right\rangle=\int_{0}^{1} d u(2 u-1)^{n} \phi_{S}^{s}(u, \mu)
\end{array}
$$

From the orthogonal relation Eq.(10) the Gegenbauer moments $a_{n}$ can be expressed in terms of $\left\langle\xi^{n}\right\rangle$, for our purpose

$$
a_{2}=\frac{5}{2}\left(3\left\langle\xi^{2}\right\rangle-1\right), \quad a_{4}=\frac{9}{8}\left(35\left\langle\xi^{4}\right\rangle-30\left\langle\xi^{2}\right\rangle+3\right) .
$$

The next step is to calculate the so-called moments appearing in Eq.(11), to this end we consider the following two-point correlation function with derivatives

$$
\begin{aligned}
I_{n 0}(z, q) & =i \int d^{4} x e^{i q x}\left\langle 0\left|T O_{n}(x) O^{\dagger}(0)\right| 0\right\rangle \\
& =(z \cdot q)^{n} I_{n 0}^{\mathrm{OPE}}\left(q^{2}\right)
\end{aligned}
$$

with

$$
O_{n}(x)=\bar{q}_{1}(x)(i z \cdot \overleftrightarrow{D})^{n} q_{2}(x), \quad O^{\dagger}(0)=\bar{q}_{2}(0) q_{1}(0)
$$

The above correlation function can be expressed in terms of the operator product expansion. Up to leading order of $\alpha_{s}$ and dimension-six we get $^{2}$

$$
\begin{aligned}
I_{n 0}(z, q) & =(z \cdot q)^{n}\left[-\frac{3}{8 \pi^{2}} \frac{1}{n+1} q^{2} \ln \frac{-q^{2}}{\mu^{2}}+\frac{3+n}{24}\left\langle\frac{\alpha_{s}}{\pi} G^{2}\right\rangle\right. \\
& -\frac{1}{q^{2}}\left(\frac{n+1}{2} m_{1}+m_{2}\right)\left\langle\bar{q}_{1} q_{1}\right\rangle-\frac{1}{q^{2}}\left(m_{1}+\frac{n+1}{2} m_{2}\right)\left\langle\bar{q}_{2} q_{2}\right\rangle \\
& -\frac{1}{2 q^{4}} m_{2}\left\langle g_{s} \bar{q}_{1} \sigma G q_{1}\right\rangle-\frac{1}{2 q^{4}} m_{1}\left\langle g_{s} \bar{q}_{2} \sigma G q_{2}\right\rangle \\
& +\frac{4 \pi}{27} \frac{\alpha_{s}}{q^{4}}\left(n^{2}+3 n-4\right)\left(\left\langle\bar{q}_{1} q_{1}\right\rangle^{2}+\left\langle\bar{q}_{2} q_{2}\right\rangle^{2}\right) \\
& \left.-\frac{48}{9} \frac{\alpha_{s}}{q^{4}}\left\langle\bar{q}_{1} q_{1}\right\rangle\left\langle\bar{q}_{2} q_{2}\right\rangle\right] .
\end{aligned}
$$

with $n$ is even thus only the scalar even moments exist. This is the theoretical side of the correlation function from the quark-gluon dynamics. On the other hand Eq.(14) can also be

\footnotetext{
${ }^{2}$ Noticing the operator product expansion is different from Ref. [31] on the mass-dependent condensates terms, but there is little impact on the results since these terms are greatly suppressed by the quark mass. We find the operator product expansion in Eq. (15) presents a well extremum behavior.
} 
derived phenomenologically based on the dispersion relation

$$
I_{n 0}\left(q^{2}\right)=\frac{1}{\pi} \int_{0}^{\infty} d s \frac{\operatorname{Im} I_{n 0}^{p h}(s)}{s-q^{2}}+\text { subtr. const. }
$$

The imaginary part $\operatorname{Im} I_{n 0}^{p h}(s)$ is obtained by inserting a complete quantum sets $\sum|n\rangle\langle n\rangle$ into Eq.(14) which reads

$$
\operatorname{Im} I_{n 0}^{p h}\left(q^{2}\right)=\pi m_{S}^{2} f_{S}^{2}\left\langle\xi^{n}\right\rangle \delta\left(q^{2}-m_{S}^{2}\right)+\frac{3}{8 \pi^{2}} \frac{1}{n+1} \pi q^{2} \theta\left(q^{2}-s_{0}\right),
$$

By equating the theoretical and phenomenological sides of $I_{n 0}(z, q)$ we get the sum rules

$$
I_{n 0}^{O P E}\left(q^{2}\right)=\frac{1}{\pi} \int_{0}^{\infty} d s \frac{\operatorname{Im} I_{n 0}^{p h}(s)}{s-q^{2}}+\text { subtr. const. }
$$

Substituting Eq.(15) and Eq.(17) into Eq.(18), taking Borel transformation and subtracting the continuum contributions we arrive the desired scalar moment sum rules

$$
\begin{aligned}
m_{S}^{2} f_{S}^{2}\left\langle\xi_{s}^{n}\right\rangle \exp \left[-\frac{m_{S}^{2}}{M^{2}}\right] & =\frac{3}{8 \pi^{2}} \frac{1}{n+1} \int_{0}^{s_{0}} d s s \exp \left[-\frac{s}{M^{2}}\right]+\frac{3+n}{24}\left\langle\frac{\alpha_{s}}{\pi} G^{2}\right\rangle \\
& +\left(\frac{n+1}{2} m_{1}+m_{2}\right)\left\langle\bar{q}_{1} q_{1}\right\rangle+\left(m_{1}+\frac{n+1}{2} m_{2}\right)\left\langle\bar{q}_{2} q_{2}\right\rangle \\
& -\frac{1}{2 M^{2}} m_{2}\left\langle g_{s} \bar{q}_{1} \sigma G q_{1}\right\rangle-\frac{1}{2 M^{2}} m_{1}\left\langle g_{s} \bar{q}_{2} \sigma G q_{2}\right\rangle \\
& +\frac{4 \pi}{27} \frac{\alpha_{s}}{M^{2}}\left(n^{2}+3 n-4\right)\left[\left\langle\bar{q}_{1} q_{1}\right\rangle^{2}+\left\langle\bar{q}_{2} q_{2}\right\rangle^{2}\right] \\
& -\frac{48 \pi}{9} \frac{\alpha_{s}}{M^{2}}\left\langle\bar{q}_{1} q_{1}\right\rangle\left\langle\bar{q}_{2} q_{2}\right\rangle .
\end{aligned}
$$

Up to now all our analysis still confines in the conventional QCD sum rules, in the coming subsection the instanton will take part in the game.

\section{B. Inclusion of the instanton effects in moment sum rules}

In this subsection our calculation are in four-dimension Euclidean space unless explicitly point out. Instanton is the nontrivial solution of classical field equation in four-dimension Euclidean gauge-field theories which is first discovered by Belavin et al [10]. Subsequently 't Hooft[12] derived the instanton with topological charge $Q=1$ in four-dimension Euclidean space

$$
\begin{aligned}
A_{\mu}^{a}(x) & =\frac{2}{g} \eta_{a \mu \nu} \frac{\left(x-x_{0}\right)_{\nu}}{\left(x-x_{0}\right)^{2}+\rho^{2}} \\
G_{\mu \nu}^{a}(x) & =-\frac{4}{g} \eta_{a \mu \nu} \frac{\rho^{2}}{\left[\left(x-x_{0}\right)^{2}+\rho^{2}\right]^{2}}
\end{aligned}
$$


where $\rho$ is instanton size, $\eta_{a \mu \nu}$ is the t'Hooft $\eta$ symbol, $x_{0}$ is an any point in four-dimension Euclidean space called instanton center. In this instanton background field there is quark zero-mode which represent the tunneling effects, for our purpose we write the quark zero-mode propagator explicitly in regular gauge $e^{3}$

$$
\begin{aligned}
S^{z m}\left(x, y ; x_{0}\right) & =\frac{\rho^{2}}{8 \pi^{2} m^{*}} \frac{1}{\left[\left(x-x_{0}\right)^{2}+\rho^{2}\right]^{3 / 2}} \frac{1}{\left[\left(y-x_{0}\right)^{2}+\rho^{2}\right]^{3 / 2}} \\
& \times\left[\gamma_{\mu} \gamma_{\nu} \frac{1}{2}\left(1-\gamma_{5}\right)\right] \otimes\left[\tau_{\mu}^{+} \tau_{\nu}^{-}\right]
\end{aligned}
$$

where $m^{*}$ is the effective mass and

$$
\tau_{\mu}^{ \pm}=(\boldsymbol{\tau}, \pm i), \quad \boldsymbol{\tau}=\boldsymbol{\sigma}
$$

with the useful relations

$$
\begin{aligned}
\tau^{a} \tau^{b} & =\delta^{a b}+i \varepsilon^{a b c} \tau^{c}, \\
\tau_{\mu}^{+} \tau_{\nu}^{-} & =\delta_{\mu \nu}+i \eta_{a \mu \nu} \tau^{a}, \\
\tau_{\mu}^{-} \tau_{\nu}^{+} & =\delta_{\mu \nu}+i \bar{\eta}_{a \mu \nu} \tau^{a} .
\end{aligned}
$$

The instanton contribution to the scalar moment sum rules is obtained by substituting the zero-mode propagator Eq.(22) into correlation function Eq.(14)

$$
\begin{aligned}
\int d^{4} x e^{i Q x}\left\langle 0\left|T O_{n}(x) O^{\dagger}(0)\right| 0\right\rangle & =\frac{8}{\pi^{4} m_{1}^{*} m_{2}^{*}} \int d^{4} x e^{i Q x} \int d \rho n(\rho) \rho^{4} \int d^{4} x_{0} \frac{1}{\left(x_{0}^{2}+\rho^{2}\right)^{3}} \\
& \times \frac{1}{\left[\left(x-x_{0}\right)^{2}+\rho^{2}\right]^{3 / 2}}\left(i z \cdot \overleftrightarrow{D}_{I}\right)^{n} \frac{1}{\left[\left(x-x_{0}\right)^{2}+\rho^{2}\right]^{3 / 2}}
\end{aligned}
$$

with

$$
O_{n}(x)=\bar{q}_{10}(x)\left(i z \cdot \overleftrightarrow{D}_{I}\right)^{n} q_{20}(x), \quad O^{\dagger}(0)=\bar{q}_{20}(0) q_{10}
$$

where the integration over collective coordinates of instanton is explicit, the anti-instanton contribution as well as traces over $\gamma$ and $\mathrm{SU}(2)$ matrix are completed implicitly. The instanton density has the simple form proposed in[15]

$$
n(\rho)=n_{c} \delta\left(\rho-\rho_{c}\right)
$$

\footnotetext{
${ }^{3}$ For the propagator in singular gauge on can refer to 33].
} 
But now one should notice that the covariant derivative in Eq.(25) contains the instanton background field $A_{\mu}^{(I)}$ to guarantee gauge invariance

$$
D_{\mu}^{I}=\partial_{\mu}-i g A_{\mu}^{(I) a} t^{a}
$$

where $t^{a}$ is the $\mathrm{SU}(2)$ generator $t^{a}=\sigma^{a} / 2$ with the normalization condition

$$
\operatorname{tr}\left[t^{a} t^{b}\right]=\frac{1}{2} \delta^{a b}
$$

Firstly we take a close look at the covariant derivative

$$
\begin{aligned}
\left(i z \cdot \overleftrightarrow{D}_{I}\right)^{n} & =\left[i z \cdot\left(\vec{D}_{I}-\overleftarrow{D}_{I}\right)\right]^{n} \\
& =\left\{i z_{\mu}\left[\left(\vec{\partial}_{\mu}-i g A_{\mu}^{(I) a} t^{a}\right)-\left(\overleftarrow{\partial}_{\mu}+i g A_{\mu}^{(I) a} t^{a}\right)\right]\right\}^{n}
\end{aligned}
$$

We can sort the terms in expansion of Eq.(29) into two kinds. The first one only contains the differential operator which acts on zero-mode propagator from left and right, the second one is the remaining parts which include all the instanton field involved terms. In following we will elucidate the complete contribution from these two kinds at $n=2$ and $n=4$ by explicit calculation.

Contribution to the correlation function of the first kind is easy to calculate such that we can derive a general formula for $n$ as follows ${ }^{4}$

$$
\begin{aligned}
\Pi_{n}^{\mathrm{first}}\left(Q^{2}\right) & =\int d^{4} x e^{i Q x}\left\langle 0\left|T O_{n}(x) O^{\dagger}(0)\right| 0\right\rangle \\
& =\frac{8 \rho^{4}}{\pi^{4} m_{1}^{*} m_{2}^{*}} \int d^{4} x_{0} e^{i Q x_{0}} \frac{1}{\left(x_{0}^{2}+\rho^{2}\right)^{3}} \times A(2 i)^{n} \\
& \times\left(-i z \cdot \frac{\partial}{\partial Q}\right)^{n} \int d^{4} x e^{i Q x} \frac{1}{\left(x^{2}+\rho^{2}\right)^{n+3}}
\end{aligned}
$$

where $A$ is constant

$$
\begin{aligned}
A & =\left[1+(-1)^{n}\right] \cdot \frac{3}{2} \cdot \frac{5}{2} \cdot \ldots \cdot \frac{2 n+1}{2} \\
& +\sum_{k=1}^{n-1} C_{n}^{k}(-1)^{n+k} \cdot \frac{3}{2} \cdot \frac{5}{2} \cdot \ldots \cdot \frac{2(n-k)+1}{2} \times \frac{3}{2} \cdot \frac{5}{2} \cdot \ldots \cdot \frac{2 k+1}{2}
\end{aligned}
$$

\footnotetext{
${ }^{4}$ Without confusion we take $\rho_{c} \rightarrow \rho$ for simplicity.
} 
It is worthy to mention that in deriving Eq.(30) from Eq.(25) the light-cone constraint $z^{2}=0$ is crucial otherwise there will be big masses. Now the remaining work is trivial with the help of the following formulae[32, 34 ]

$$
\begin{aligned}
\int d^{4} x \frac{e^{i Q x}}{\left(x^{2}+\rho^{2}\right)^{\nu}} & =\frac{2 \pi^{2}}{\Gamma(\nu)}\left(\frac{Q \rho}{2}\right)^{\nu-2} \frac{K_{2-\nu}(Q \rho)}{\rho^{2 \nu-4}} \\
\left(\frac{d}{z d z}\right)^{m}\left[z^{\nu} K_{\nu}(z)\right] & =(-)^{m} z^{\nu-m} K_{\nu-m}(z) \\
K_{-\nu}(z) & =K_{\nu}(z)
\end{aligned}
$$

where $Q^{2}=-q^{2}$ and $K_{\nu}(z)$ is the MacDonald function. When the smoke clears, we get the desired results for the first kind contribution

$$
\begin{aligned}
\Pi_{n}^{\mathrm{first}}\left(Q^{2}\right) & =\int d^{4} x e^{i Q x}\left\langle 0\left|T O_{n}(x) O^{\dagger}(0)\right| 0\right\rangle \\
& =\frac{n_{c} \rho^{2}}{\pi m_{1}^{*} m_{2}^{*}} \frac{2^{n+1}\left[1+(-1)^{n}\right](1+n)}{\Gamma(n+3)}\left[\Gamma\left(\frac{n+1}{2}\right)\right]^{2} \\
& \times(z \cdot Q)^{n} Q^{2} K_{1}^{2}(Q \rho),
\end{aligned}
$$

We find the non-vanishing contribution of the second kind for $n=2$ is

$$
\Pi_{2}^{\text {second }}\left(Q^{2}\right)=i^{2} \int d^{4} x e^{i Q x} S_{10}\left(0, x ; x_{0}\right)\left(-2 i g z \cdot A^{(I)}\right)^{2} S_{20}\left(x, 0 ; x_{0}\right),
$$

Substituting the zero-mode propagator Eq.(22) and instanton field Eq.(20) at regular gauge into Eq.(33), for definite we write its explicit form as follows

$$
\begin{aligned}
\Pi_{2}^{\text {second }}\left(Q^{2}\right) & =-\frac{4 g^{2}}{64 \pi^{4} m_{1}^{*} m_{2}^{*}} \int d^{4} x e^{i Q \cdot x} \int d \rho n(\rho) \rho^{4} \int d^{4} x_{0} \frac{1}{\left(x_{0}^{2}+\rho^{2}\right)^{3 / 2}} \\
& \times \frac{1}{\left[\left(x-x_{0}\right)^{2}+\rho^{2}\right]^{3 / 2}} \gamma_{\mu} \gamma_{\nu} \frac{1}{2}\left(1-\gamma_{5}\right) \tau_{\mu}^{+} \tau_{\nu}^{-} \\
& \times z_{\sigma} \frac{2}{g} \eta_{a \sigma \delta} \frac{\left(x-x_{0}\right)_{\delta}}{\left(x-x_{0}\right)^{2}+\rho^{2}} t^{a} z_{\rho} \frac{2}{g} \eta_{b \rho \gamma} \frac{\left(x-x_{0}\right)_{\gamma}}{\left(x-x_{0}\right)^{2}+\rho^{2}} t^{b} \\
& \times \frac{1}{\left(x_{0}^{2}+\rho^{2}\right)^{3 / 2}} \frac{1}{\left[\left(x-x_{0}\right)^{2}+\rho^{2}\right]^{3 / 2}} \gamma_{\alpha} \gamma_{\beta} \frac{1}{2}\left(1-\gamma_{5}\right) \tau_{\alpha}^{+} \tau_{\beta}^{-} \\
& =-\frac{n_{c} \rho_{c}^{4}}{64 \pi^{4} m_{1}^{*} m_{2}^{*}} \int d^{4} x e^{i Q \cdot x} \int d^{4} x_{0} \frac{1}{\left(x_{0}^{2}+\rho_{c}^{2}\right)^{3}} \frac{1}{\left[\left(x-x_{0}\right)^{2}+\rho_{c}^{2}\right]^{5}} \\
& \times z_{\sigma} \eta_{a \sigma \delta}\left(x-x_{0}\right)_{\delta} z_{\rho} \eta_{b \rho \gamma}\left(x-x_{0}\right)_{\gamma} \\
& \times \operatorname{tr}\left[\gamma_{\mu} \gamma_{\nu} \frac{1}{2}\left(1-\gamma_{5}\right) \gamma_{\alpha} \gamma_{\beta} \frac{1}{2}\left(1-\gamma_{5}\right)\right] \operatorname{tr}\left[\tau_{\mu}^{+} \tau_{\nu}^{-} \tau^{a} \tau^{b} \tau_{\alpha}^{+} \tau_{\beta}^{-}\right],
\end{aligned}
$$


After a lengthy calculation we obtain the contribution from instanton field involved part for $n=2$ in expansion ${ }^{5}$

$$
\Pi_{2}^{\text {second }}\left(Q^{2}\right)=\frac{n_{c} \rho^{2}}{6 m_{1}^{*} m_{2}^{*}}(z \cdot Q)^{2} Q^{2} K_{1}^{2}(Q \rho) .
$$

where the isospin dependence have been included. One can find that this part is comparable to the first kind contribution for $n=2$.

For $n=4$ the situation is more complicated when we expand Eq.(29) order by order since the differential operator $\partial_{\mu}$ can also act on instanton field and give non-vanishing contribution. In considering this effects on instanton field we find the following terms for the second kind contribution to correlator

$$
\begin{aligned}
\Pi_{4}^{\text {second }}\left(Q^{2}\right) & =i^{4} \int d^{4} x e^{i Q x}\left\{S_{10}\left(0, x ; x_{0}\right) 4[z \cdot(\vec{\partial}-\overleftarrow{\partial})]^{2} S_{20}\left(x, 0 ; x_{0}\right)\right\}\left(-2 i g z \cdot A^{(I)}\right)^{2} \\
& +i^{4} \int d^{4} x e^{i Q x} S_{10}\left(0, x ; x_{0}\right)\left[4 z_{\mu} z_{\nu} z_{\alpha} z_{\beta} A_{\alpha}^{(I)}\left(\partial_{\mu} \partial_{\nu} A_{\beta}^{(I)}\right)(-2 i g)^{2}\right. \\
& \left.+\left(-2 i g z \cdot A^{(I)}\right)^{4}\right] S_{20}\left(x, 0 ; x_{0}\right)
\end{aligned}
$$

where for brevity the $\mathrm{SU}(2)$ generator index is suppressed. Some effort later we obtain

$$
\Pi_{4}^{\text {second }}\left(Q^{2}\right)=\frac{3 n_{c} \rho^{2}}{20 m_{1}^{*} m_{2}^{*}}(z \cdot Q)^{4} Q^{2} K_{1}^{2}(Q \rho) .
$$

Notice that we have included the anti-instanton effects both in Eq.(35) and Eq.(37). Combining Eq.(32), Eq.(35) and Eq.(37) we get the complete zero-mode contribution to correlation function

$$
\Pi_{2}^{\mathrm{zm}}\left(Q^{2}\right)=\Pi_{2}^{\mathrm{first}}\left(Q^{2}\right)+\frac{n_{c} \rho^{2}}{6 m_{1}^{*} m_{2}^{*}}(z \cdot Q)^{2} Q^{2} K_{1}^{2}(Q \rho),
$$

for $n=2$ and

$$
\Pi_{4}^{\mathrm{zm}}\left(Q^{2}\right)=\Pi_{4}^{\mathrm{first}}\left(Q^{2}\right)+\frac{3 n_{c} \rho^{2}}{20 m_{1}^{*} m_{2}^{*}}(z \cdot Q)^{4} Q^{2} K_{1}^{2}(Q \rho),
$$

for $n=4$, from the two equations above one can find the contribution of two kinds is comparable thus we can not omit one of them simply.

After dealing with the quark zero-mode in the instanton background field, now we calculate non-zero mode contribution to the correlation function. For $n=2$ this part is

$$
\Pi_{2}^{\mathrm{nzm}}\left(Q^{2}\right)=i^{2} \int d^{4} x e^{i Q x} S_{1}^{\mathrm{nzm}}(0, x)\left(-2 i g z \cdot A^{(I)}\right)^{2} S_{2}^{\mathrm{nzm}}(x, 0),
$$

\footnotetext{
${ }^{5}$ For simplicity we replace $\rho_{c}$ by $\rho$.
} 
where $S_{1}^{\mathrm{nzm}}(0, x)$ and $S_{2}^{\mathrm{nzm}}(x, 0)$ are the non-zero-mode propagators. In instanton background field the complete form of quark propagator consists of zero-mode and non-zero-mode parts

$$
S_{I}(x, y)=S_{I}^{\mathrm{zm}}(x, y)+S_{I}^{\mathrm{nzm}}(x, y),
$$

In previous paragraphs we have completed all contribution to correlation function from zeromode in single instanton approximation. To obtain the non-zero-mode contribution we need $S_{I}^{\mathrm{nzm}}(x, y)$, while $S_{I}^{\mathrm{nzm}}(x, y)$ is a quite complicated object. It was shown[49]51] that it is reliable to take massless free propagator approximation for $S_{I}^{\mathrm{nzm}}(x, y)$ if the zero-mode contribution to Green function is maximal. Fortunately in present calculation this requirement can be met since there is direct instaton contribution to the correlation function we considered thus it is convenient to take massless free propagator approximation

$$
S_{I}^{\mathrm{nzm}}(x, y)=\frac{1}{2 \pi^{2}} \frac{\not x-\not y}{(x-y)^{4}}
$$

for non-zero-mode. At this point it is necessary to stress that in vector channel this approximation is no longer valid otherwise the vector current is not always conserved[52]. In this case the propagator of non-zero-mode is very involved one can refer to Ref.[16] for details.

Combining Eq.(40) and Eq.(42) we arrive

$$
\Pi_{2}^{\mathrm{nzm}}\left(Q^{2}\right)=\frac{8 n_{c}}{\pi^{2}} \int d^{4} x e^{i Q x} \frac{1}{x^{6}} \int d^{4} x_{0} \frac{\left[z \cdot\left(x-x_{0}\right)\right]^{2}}{\left[\left(x-x_{0}\right)^{2}+\rho^{2}\right]^{2}},
$$

According to translation invariance $\left(x-x_{0}\right) \rightarrow u$ we can factorize out the the integral with respect to instanton center $x_{0}$ thus there are two separate integral given by nonzero mode and instanton field. Since $z^{2}=0$ it is easy to see the instanton integral is vanishing therefore there is no contribution of nonzero mode to correlation function. In other word nonzero mode and instanton field do not "entangle" each other. The physical meaning is that in this case the instanton field does not transfer momentum from one quark to another. Similar analysis also hold for $n=4$.

To be consistent with Eq.(19) we should reformulate the total instanton induced contributions in term of dispersion relation. For this purpose noticing the properties of MacDonald function under analytical continuation are 18, 35]

$$
K_{\nu}(z)= \begin{cases}\frac{i \pi}{2} e^{i \pi \nu / 2} H_{\nu}^{(1)}\left(z e^{i \pi / 2}\right) & -\pi<\arg z \leq \frac{\pi}{2} \\ -\frac{i \pi}{2} e^{-i \pi \nu / 2} H_{\nu}^{(1)}\left(z e^{-i \pi / 2}\right) & \frac{\pi}{2}<\arg z \leq \pi\end{cases}
$$


in above expression $H_{\nu}^{(1)}(z)$ is the Hankel function of the first kind

$$
H_{\nu}^{(1)}(z)=J_{\nu}(z)+i Y_{\nu}(z)
$$

where $J_{\nu}(z)$ and $Y_{\nu}(z)$ are the Bessel functions and Neumann functions, respectively. The last step is improving Eq.(32) so that it is consistent with Eq.(19). To this end noticing the cut structure of the Hankel functions Eq.(45) one can find

$$
\operatorname{Im} K_{1}^{2}(-i \rho \sqrt{s})=\frac{\pi^{2}}{2} J_{1}(\rho \sqrt{s}) Y_{1}(\rho \sqrt{s})+\text { singular term, }
$$

As usual in terms of the dispersion relation we get the final results improved by the Borel transformation and continuum contribution subtracted. To be definite we collect the whole results as follows

$$
\begin{aligned}
m_{S}^{2} f_{S}^{2}\left\langle\xi_{s}^{2}\right\rangle \exp \left[-\frac{m_{S}^{2}}{M^{2}}\right] & =\Pi_{2}^{O P E}\left(s_{0}, M^{2}\right) \\
& +(-1)^{I} \frac{\pi n_{c} \rho^{2}}{3 m_{1}^{*} m_{2}^{*}} \int_{0}^{s_{0}} d s s J_{1}(\rho \sqrt{s}) Y_{1}(\rho \sqrt{s}) \exp \left[-\frac{s}{M^{2}}\right]
\end{aligned}
$$

for $\left\langle\xi_{s}^{2}\right\rangle$ and

$$
\begin{aligned}
m_{S}^{2} f_{S}^{2}\left\langle\xi_{s}^{4}\right\rangle \exp \left[-\frac{m_{S}^{2}}{M^{2}}\right] & =\Pi_{4}^{O P E}\left(s_{0}, M^{2}\right) \\
& +(-1)^{I} \frac{8 \pi n_{c} \rho^{2}}{45 m_{1}^{*} m_{2}^{*}} \int_{0}^{s_{0}} d s s J_{1}(\rho \sqrt{s}) Y_{1}(\rho \sqrt{s}) \exp \left[-\frac{s}{M^{2}}\right],
\end{aligned}
$$

for $\left\langle\xi_{s}^{4}\right\rangle$ where the isospin dependence has been considered and $\Pi_{n}^{O P E}$ is the right hand side of Eq.(19). It is easy to derive the pseudoscalar moment sum rules from the scalar ones if we consider the effects of $i \gamma_{5}$ carefully in calculations

$$
\begin{aligned}
\frac{m_{P}^{4} f_{P}^{2}\left\langle\xi_{p}^{2}\right\rangle}{\left(m_{1}+m_{2}\right)^{2}} \exp \left[-\frac{m_{P}^{2}}{M^{2}}\right] & =\Pi_{2}^{p s, O P E}\left(s_{0}, M^{2}\right) \\
& -(-1)^{I} \frac{\pi n_{c} \rho^{2}}{3 m_{1}^{*} m_{2}^{*}} \int_{0}^{s_{0}} d s s J_{1}(\rho \sqrt{s}) Y_{1}(\rho \sqrt{s}) \exp \left[-\frac{s}{M^{2}}\right],
\end{aligned}
$$

for $\left\langle\xi_{p}^{2}\right\rangle$ and

$$
\begin{aligned}
\frac{m_{P}^{4} f_{P}^{2}\left\langle\xi_{p}^{4}\right\rangle}{\left(m_{1}+m_{2}\right)^{2}} \exp \left[-\frac{m_{P}^{2}}{M^{2}}\right] & =\Pi_{4}^{p s, O P E}\left(s_{0}, M^{2}\right) \\
& -(-1)^{I} \frac{8 \pi n_{c} \rho^{2}}{45 m_{1}^{*} m_{2}^{*}} \int_{0}^{s_{0}} d s s J_{1}(\rho \sqrt{s}) Y_{1}(\rho \sqrt{s}) \exp \left[-\frac{s}{M^{2}}\right],
\end{aligned}
$$


for $\left\langle\xi_{p}^{4}\right\rangle$ where $\Pi_{n}^{p s, O P E}$ represents the following instanton-free sum rules

$$
\begin{aligned}
\Pi_{n}^{p s, O P E}\left(s_{0}, M^{2}\right) & =\frac{3}{8 \pi^{2}} \frac{1}{n+1} \int_{0}^{s_{0}} d s s \exp \left[-\frac{s}{M^{2}}\right]+\frac{n-3}{24}\left\langle\frac{\alpha_{s}}{\pi} G^{2}\right\rangle \\
& +\left(\frac{n+1}{2} m_{1}-m_{2}\right)\left\langle\bar{q}_{1} q_{1}\right\rangle+\left(-m_{1}+\frac{n+1}{2} m_{2}\right)\left\langle\bar{q}_{2} q_{2}\right\rangle \\
& +\frac{1}{2 M^{2}} m_{2}\left\langle g_{s} \bar{q}_{1} \sigma G q_{1}\right\rangle+\frac{1}{2 M^{2}} m_{1}\left\langle g_{s} \bar{q}_{2} \sigma G q_{2}\right\rangle \\
& +\frac{4 \pi}{27} \frac{\alpha_{s}}{M^{2}}\left(n^{2}+3 n-4\right)\left[\left\langle\bar{q}_{1} q_{1}\right\rangle^{2}+\left\langle\bar{q}_{2} q_{2}\right\rangle^{2}\right] \\
& +\frac{48 \pi}{9} \frac{\alpha_{s}}{M^{2}}\left\langle\bar{q}_{1} q_{1}\right\rangle\left\langle\bar{q}_{2} q_{2}\right\rangle .
\end{aligned}
$$

Obviously the instanton contribution in pseudoscalar channel is opposite to scalar one which reflects the chirality-dependence of instanton effects.

Now all the formulae needed have been fixed. The parameters which will be adopted in our numerical analysis are as follows [6, 36, 37]

$$
\begin{gathered}
\alpha_{s}=0.517, \quad\left\langle\frac{\alpha_{s}}{\pi} G^{2}\right\rangle=0.012 \pm 0.006 \mathrm{GeV}^{4}, \\
\langle\bar{u} u\rangle=\langle\bar{d} d\rangle=-(0.225 \pm 0.15)^{3} \mathrm{GeV}^{3}, \quad\langle\bar{s} s\rangle=(0.8 \pm 0.2)\langle\bar{u} u\rangle \\
m_{u}=0.004 \mathrm{GeV}, m_{d}=0.006 \mathrm{GeV}, \quad m_{s}=0.12 \mathrm{GeV}, \\
\left\langle g_{s} \bar{u} \sigma G u\right\rangle=\left\langle g_{s} \bar{d} \sigma G d\right\rangle=0.8 \mathrm{GeV}^{2}\langle\bar{u} u\rangle, \quad\left\langle g_{s} \bar{s} \sigma G s\right\rangle=0.8\left\langle g_{s} \bar{u} \sigma G u\right\rangle .
\end{gathered}
$$

We read the masses and decay constants of $f_{0}, K_{0}^{*}$ and $a_{0}$ from [19]

$$
\begin{gathered}
m_{f_{0}}=1380 \mathrm{MeV}, f_{f_{0}}=375 \mathrm{MeV} \\
m_{K_{0}^{*}}=1450 \mathrm{MeV}, f_{K_{0}^{*}}=370 \mathrm{MeV} \\
m_{a_{0}}=1480 \mathrm{MeV}, \quad f_{a_{0}}=370 \mathrm{MeV} .
\end{gathered}
$$

The mass and decay constant pion are[37]

$$
m_{\pi}=140 \mathrm{MeV}, \quad f_{\pi}=130 \mathrm{MeV} .
$$

All the parameters in Eq.(51) as well as Eq.(52) and Eq.(153) are taken at $\mu=1 \mathrm{GeV}$.

The remaining important parameters are the instanton related ones. For the effective masses 
of quarks and instanton size the following values are work well[19, 33]

$$
\begin{array}{r}
m_{u}^{*}=m_{d}^{*}=86 \mathrm{MeV}, \\
m_{s}^{*}=114 \pm 28 \mathrm{MeV}, \\
\rho=\frac{1}{3} \mathrm{fm}=\frac{1}{0.6} \mathrm{GeV}^{-1} .
\end{array}
$$

The last parameter is the instanton density which still needs improvement. The original value $n_{c}=\frac{1}{2} \mathrm{fm}^{-4}$ is used widely [15, 38, 39], while the lattice calculation suggested $n_{c} \sim 1 \mathrm{fm}{ }^{-4}[40]$. The work of Cristoforetti et al [41] based on the interacting instanton liquid model shown even a larger one was needed, i.e. $n_{c}=3 \mathrm{fm}^{-4}$, to reproduce the nucleon mass and the low-energy constants in chiral perturbation theory. For this reason we will investigate the sensitivity of the moments consequently the LCDAs for different instanton density.

\section{RESULTS AND DISCUSSIONS}

Firstly we present the selection rule of the threshold and Borel window. The continuum(and exited states) as well as dimension-six condensates contribution should be controllable, as usual [8, 31, 47, 48] we demand that in the instanton-free sum rules the continuum contribution the part in the dispersive integral from $s_{0}$ to $\infty$ should be less than $30 \%$ the total perturbative dispersion integration which sets an upper limit to us, the dimension-six condensates be no more than $15 \%$ which sets an lower limit to us. If there is extremum within the Borel window selected, we take it as our calculated value otherwise the mid-value within the window will be adopted. Then we turn on instanton contribution under same threshold and Borel window since in this way there will be well comparison for the two cases. Of course one can analyze the moments by separate threshold and Borel window when instanton effect turn on, however in general the sum rule is sensitive to threshold and Borel window that the instanton effect may be smeared by the change inducing by the new threshold and Borel window. In following discussions all the Borel windows satisfy the selection rule unless explicitly state.

Along with the steps we find for pion the moment $\left\langle\xi_{p}^{2}\right\rangle$ at threshold $s_{0}=4.0 \pm 0.2 \mathrm{GeV}^{2}$ and Borel window $M^{2} \in[1.35,1.65] \mathrm{GeV}^{2}$ as well as $\left\langle\xi_{p}^{4}\right\rangle$ at $s_{0}=4.4 \pm 0.2 \mathrm{GeV}^{2}$ and $M^{2} \in$ $[1.2,1.5] \mathrm{GeV}^{2}$ are stable from sum rules Eq.(49) and Eq.(50) at $n_{c}=0$ respectively. The midvalue are $\left\langle\xi_{p}^{2}\right\rangle=0.34$ and $\left\langle\xi_{p}^{4}\right\rangle=0.21$ which are shown in Fig.1. When turning on the instanton 
effects we find $\left\langle\xi_{p}^{2}\right\rangle=0.52$ and $\left\langle\xi_{p}^{4}\right\rangle=0.36$ at $n_{c}=\frac{1}{2} \mathrm{fm}^{-4}$. If increasing instanton density, for instance at $n_{c}=1 \mathrm{fm}^{-4}$ we find $\left\langle\xi_{p}^{2}\right\rangle=0.71$ and $\left\langle\xi_{p}^{4}\right\rangle=0.50$. Obviously the moments increase with instanton density increasing. Hence it is expected that at some larger $n_{c}$ the second moment will be more than one, for instance if we take $n_{c}=2 \mathrm{fm}^{-4}$ as proposed in[41] we find $\left\langle\xi_{p}^{2}\right\rangle=1.10$ and $\left\langle\xi_{p}^{4}\right\rangle=0.80$, it is astonishing that the second moment is more than 1 ! This result is unnatural since it is obvious from Eq.(12) if assuming positive-definite LCDAs we have

$$
\left\langle\xi^{m}\right\rangle=\int_{0}^{1} d u(2 u-1)^{m} \phi(u, \mu)<\left\langle\xi^{n}\right\rangle=\int_{0}^{1} d u(2 u-1)^{n} \phi(u, \mu)<1,
$$

where

$$
n, m=2,4, \ldots ., m>n
$$

But the LCDAs themselves are not measurable, to observe their exact role we should convolute them with the hard scattering amplitudes $T$ in exclusive processes. In fact the twist-2 LCDAs of $f_{0}(980)$ given in [5] from CZ method, twist-3 ones of pion [30] as well as the work in Ref. [42 [46] show non-positive-definite behavior within some range of momentum fraction. In considering this it seems that our results may indicate non-positive-definite LCDAs. Indeed the instantoninvolved LCDAs of pion show this property as presented in Fig. 3 although the moments are still well convergent. In considering the exact form of LCDAs and instanton density are not well known nowadays so that the impact of high instanton density to LCDAs by the CZ method seems to need further study. Our results show that it seems that the sum rules Eq.(49) and Eq.(50) do not allow too large instanton density in order to get convergent moments. The obtained LCADs of pion for different instanton density is shown in the left panel of Fig. 3 ,

Then we turn to analyze $f_{0}(1370)$ with the quark content assigned in Eq.(1). The sum rules of this member is very similar to the pion except some different condensates terms induced by chirality. We can see the instanton contribution of $f_{0}(1370)$ is same as pion since there are simultaneous changes in its isospin and chirality relative to pion. At $n_{c}=0$ we get the threshold and Borel window for $\left\langle\xi_{f_{0}}^{2}\right\rangle$ and $\left\langle\xi_{f_{0}}^{4}\right\rangle$ are $s_{0}=4.7 \pm 0.2 \mathrm{GeV}^{2}, M^{2} \in[1.3,1.7] \mathrm{GeV}^{2}$ and $s_{0}=4.8 \pm 0.2 \mathrm{GeV}^{2}, M^{2} \in[1.8,2.2] \mathrm{GeV}^{2}$, respectively. Under the threshold and window there are well extremum behavior both for $\left\langle\xi_{f_{0}}^{2}\right\rangle$ and $\left\langle\xi_{f_{0}}^{4}\right\rangle$ which are shown in Fig. 2. The extremum within the range of threshold are very stable we obtain $\left\langle\xi_{f_{0}}^{2}\right\rangle=0.35,\left\langle\xi_{f_{0}}^{4}\right\rangle=0.24$. When the instanton effects turn on we find the mid-value are $\left\langle\xi_{f_{0}}^{2}\right\rangle=0.55$ and $\left\langle\xi_{f_{0}}^{4}\right\rangle=0.34$ for $n_{c}=\frac{1}{2} \mathrm{fm}^{-4}$ as well as $\left\langle\xi_{f_{0}}^{2}\right\rangle=0.76$ and $\left\langle\xi_{f_{0}}^{4}\right\rangle=0.44$ for $n_{c}=1 \mathrm{fm}^{-4}$. Similar to the case of 

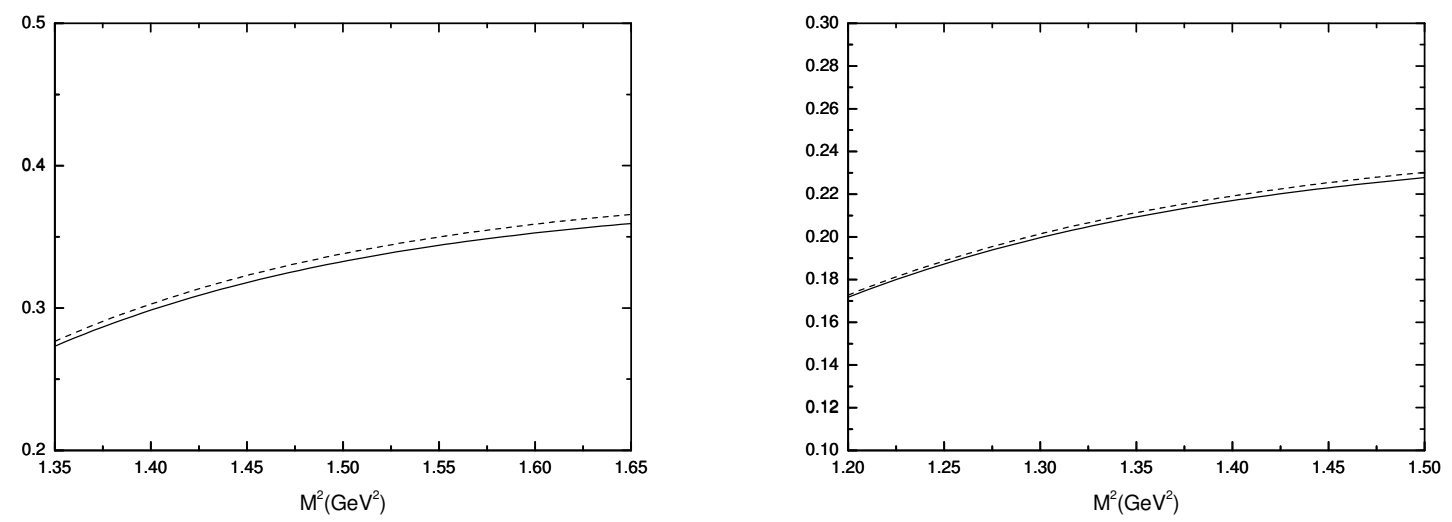

FIG. 1: Moments $\left\langle\xi_{p}^{2}\right\rangle$ (left panel) and $\left\langle\xi_{p}^{4}\right\rangle$ (right panel) of pion from instanton-free sum rules. Solid lines correspond to the central value of threshold while the dashed lines represent the threshold increasing by $0.1 \mathrm{GeV}^{2}$ relative to central value(the same for Fig.2, Fig.4 and Fig.5).
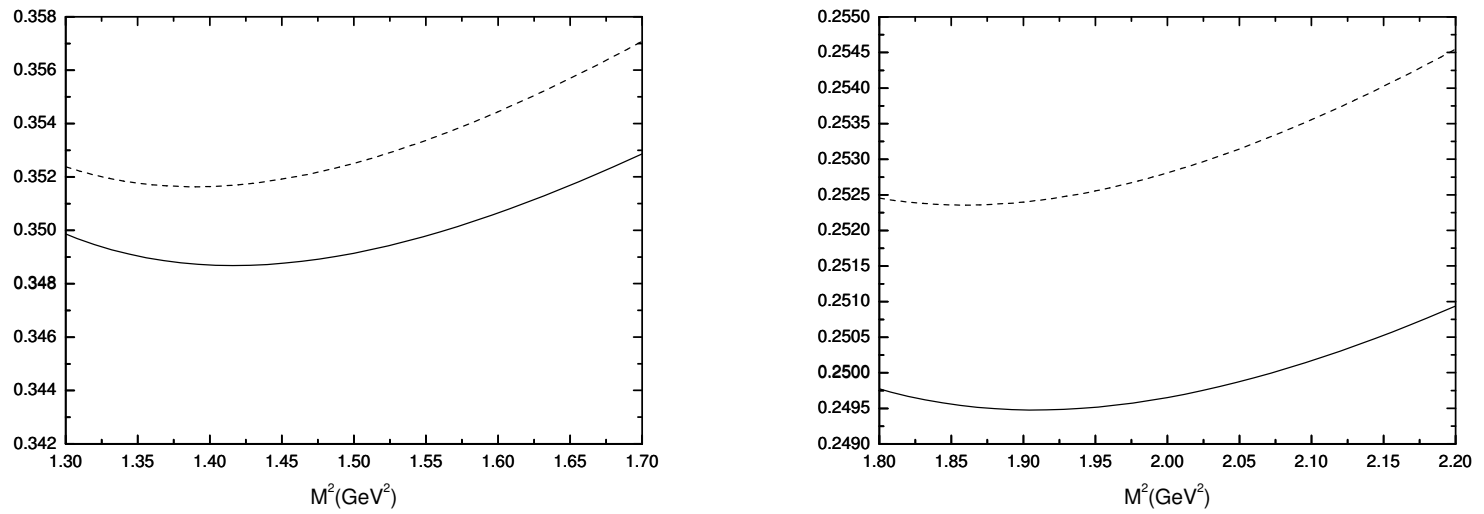

FIG. 2: Moments $\left\langle\xi_{s}^{2}\right\rangle$ (left panel) and $\left\langle\xi_{s}^{4}\right\rangle$ (right panel) of $f_{0}(1370)$ at $\mu=1 \mathrm{GeV}$ from instanton-free sum rules.

$\pi$ at $n_{c}=2 \mathrm{fm}^{-4}$ we find $\left\langle\xi_{f_{0}}^{2}\right\rangle=1.17$ which more than 1 . The moments also increase as the instanton density take a larger value which is understandable since the instanton contributions to pion and $f_{0}(1370)$ are equivalent due to the combined effects of isospin and chirality. Thus we conclude the instanton contribution is positive to the moment sum rules of pion and $f_{0}(1370)$. 

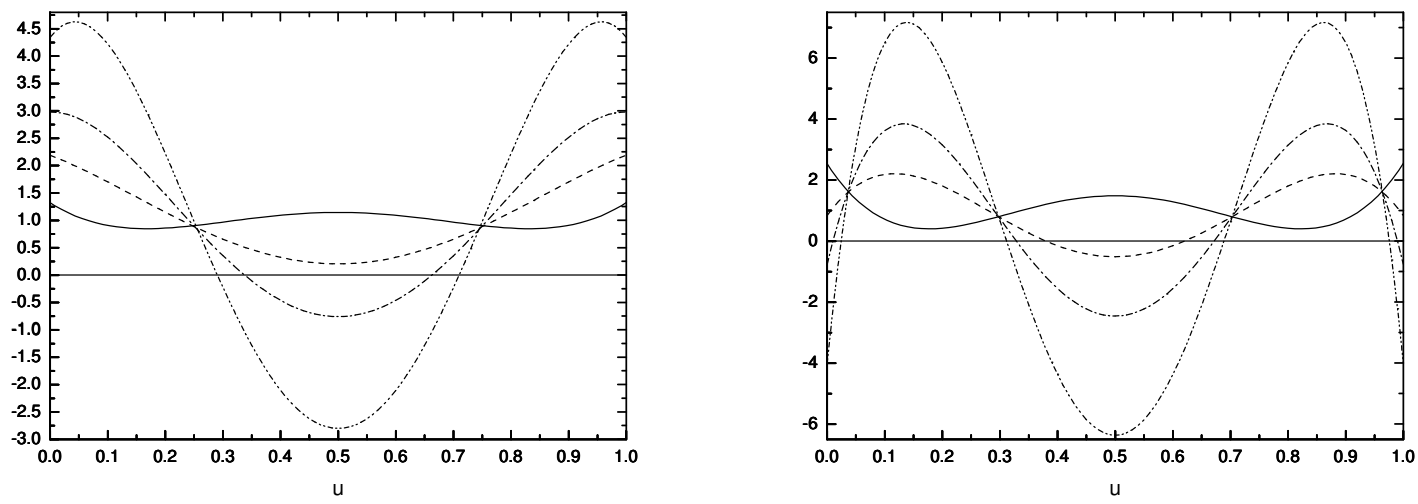

FIG. 3: Twist-3 light-cone distribution amplitudes of pion(left panel) and $f_{0}(1370)$ (right panel) as function of momentum fraction $u$ at $\mu=1 \mathrm{GeV}$ for different instanton density: solid line $n_{c}=0$, dashed $n_{c}=\frac{1}{2} \mathrm{fm}^{-4}$ and dashed-dot $n_{c}=1 \mathrm{fm}^{-4}$, dash-dot-dot $n_{c}=2 \mathrm{fm}^{-4}$ (the same for Fig.66).

The LCDAs of $f_{0}(1370)$ are plotted in Fig. 3 .

It is easy to understand the similar impact of instanton on the LCDAs of pion and $f_{0}(980)$ since the dominant parts, i.e., perturbative dispersive integral and the instanton contribution of the moment sum rules for pion and $f_{0}(1370)$ are equivalent except the chirality-dependent condensates. In fact this similarity also reflects in the LCDAs which can be observed clearly from Fig.3, One more important thing is that the LCDAs is positive-definite when there is no instanton effects while when the instanton involved there is strong impact on the profile of LCDAs. Due to the chirality-dependent parts at the two ends of momentum fraction the LCDAs of $f_{0}(1370)$ change more rapidly than pion.

The sum rules of $K_{0}^{*}(1430)$ and $a_{0}(1450)$ are nearly the same since they share same isospin and chirality in addition to the difference introduced by flavor symmetry breaking. The adopted threshold and Borel window of $\left\langle\xi_{K_{0}^{*}}^{2}\right\rangle$ and $\left\langle\xi_{K_{0}^{*}}^{4}\right\rangle$ at $n_{c}=0$ are $s_{0}=4.7 \pm 0.2 \mathrm{GeV}^{2}, M^{2} \in$ $[1.3,1.7] \mathrm{GeV}^{2}$ and $s_{0}=5.5 \pm 0.2 \mathrm{GeV}^{2}, M^{2} \in[1.35,1.75] \mathrm{GeV}^{2}$, respectively. There is little change of the extremum corresponding to different threshold both for the two moments, we get $\left\langle\xi_{K_{0}^{*}}^{2}\right\rangle=0.34$ and $\left\langle\xi_{K_{0}^{*}}^{4}\right\rangle=0.23$. When the instanton effects involved the moments change lot even at low density $n_{c}=\frac{1}{2} \mathrm{fm}^{-4}$. Both moments decrease compared with the case $n_{c}=0$, 

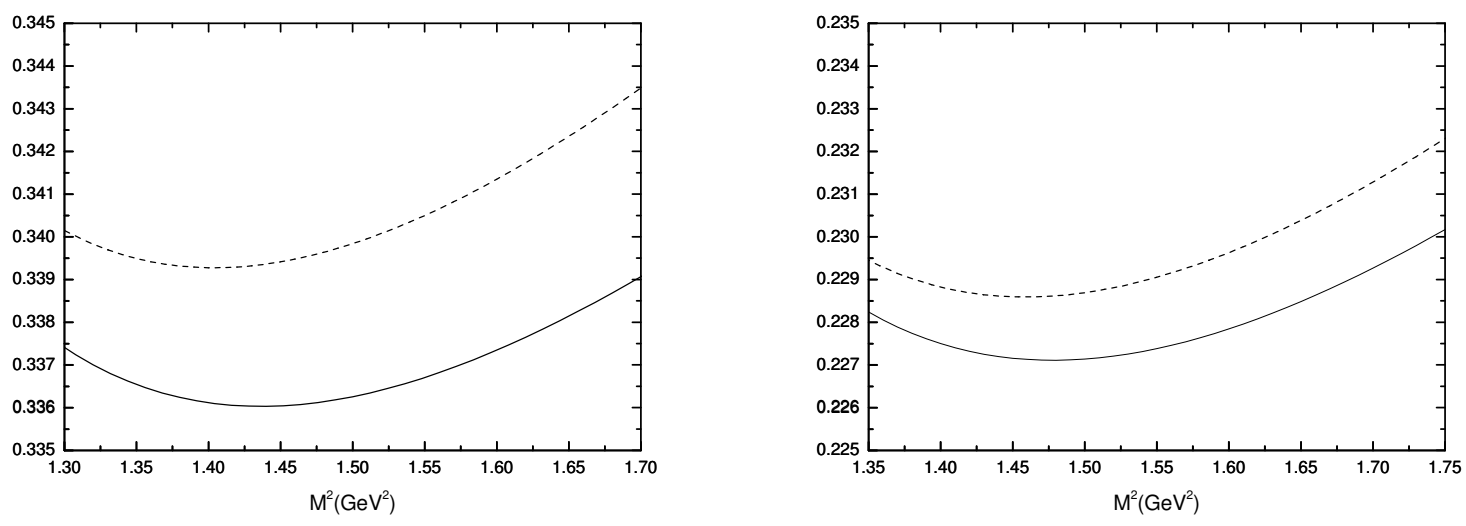

FIG. 4: Moments $\left\langle\xi_{s}^{2}\right\rangle$ (left panel) and $\left\langle\xi_{s}^{4}\right\rangle$ (right panel) of $K_{0}^{*}(1430)$ from instanton-free sum rules.
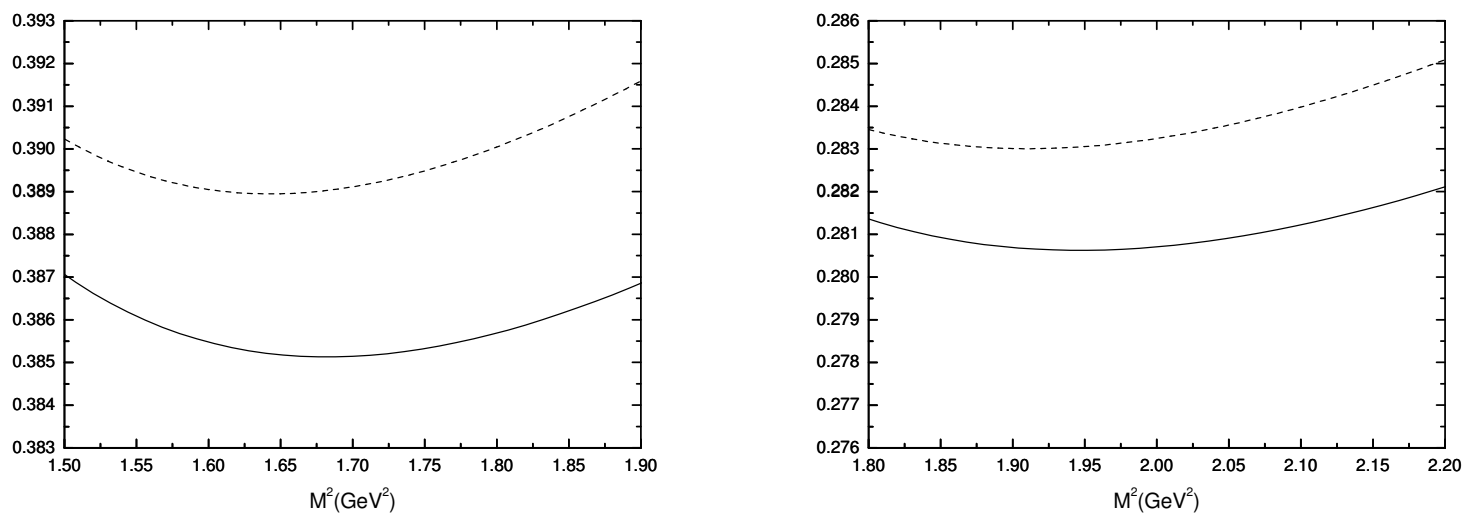

FIG. 5: Moments $\left\langle\xi_{s}^{2}\right\rangle$ (left panel) and $\left\langle\xi_{s}^{4}\right\rangle$ (right panel) of $a_{0}(1450)$ from instanton-free sum rules at $\mu=1 \mathrm{GeV}$.

we obtain $\left\langle\xi_{K_{0}^{*}}^{2}\right\rangle=0.17,\left\langle\xi_{K_{0}^{*}}^{4}\right\rangle=0.13$. If the instanton density increases further, for instance $n_{c}=1 \mathrm{fm}^{-4}$ we find there is flipping of the second and fourth moments, $\left\langle\xi_{K_{0}^{*}}^{2}\right\rangle=0.01$ and $\left\langle\xi_{K_{0}^{*}}^{4}\right\rangle=0.04$ which is unsatisfactory since it breaks the convergence. However at higher density $n_{c}=2 \mathrm{fm}^{-4}$ the convergence can recover while it develops negative value $-\left\langle\xi_{K_{0}^{*}}^{2}\right\rangle=-0.32$ and $\left\langle\xi_{K_{0}^{*}}^{4}\right\rangle=-0.16$. The LCDAs of $K_{0}^{*}(1430)$ for different instanton density are plotted in Fig.6. It is clear that the profile of LCDAs of $K_{0}^{*}(1430)$ with $n_{c} \neq 0$ just reverse to pion and $f_{0}(1370)$ 

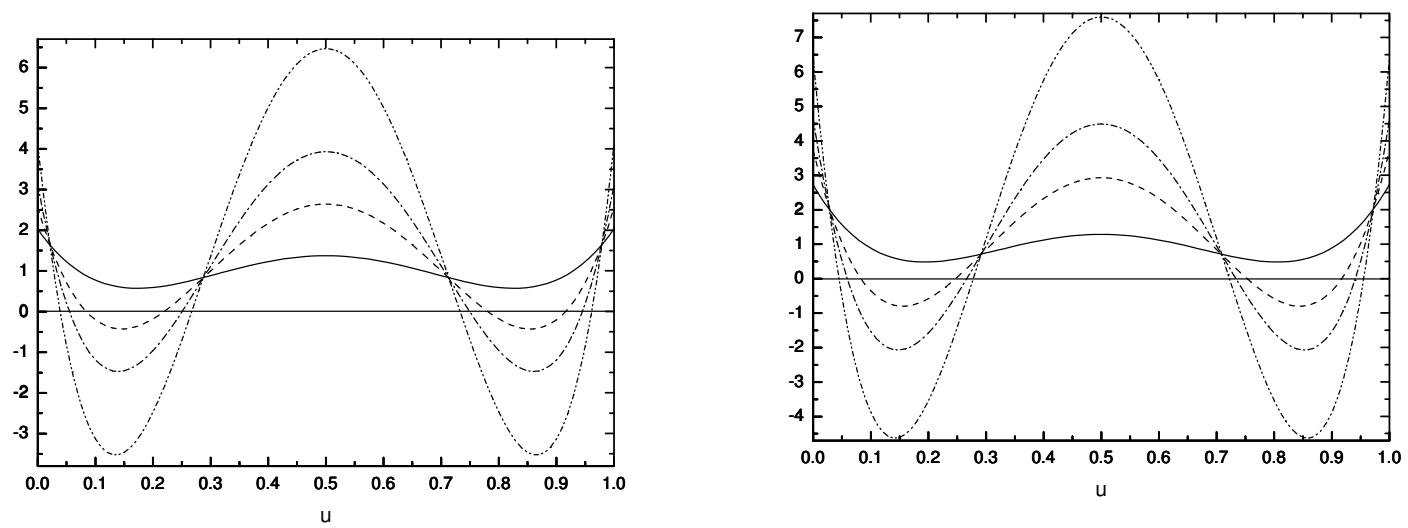

FIG. 6: Twist-3 light-cone distribution amplitudes of $K_{0}^{*}(1430)$ (left panel) and $a_{0}(1450)$ (right panel) as function of momentum fraction $u$ at $\mu=1 \mathrm{GeV}$ for different instanton density.

which well indicates the conspiracy of chirality and isospin dependence of instanton effects.

The case of $a_{0}(1450)$ runs in parallel to $K_{0}^{*}(1430)$. The threshold and working window of $\left\langle\xi_{a_{0}}^{2}\right\rangle$ and $\left\langle\xi_{a_{0}}^{4}\right\rangle$ determined from with instanton-free sum rules are $s_{0}=4.9 \pm 0.2 \mathrm{GeV}^{2}$, $M^{2} \in[1.5,1.9] \mathrm{GeV}^{2}$ and $s_{0}=5.6 \pm 0.2 \mathrm{GeV}^{2}, M^{2} \in[1.8,2.2] \mathrm{GeV}^{2}$, respectively. We obtain the extremum $\left\langle\xi_{a_{0}}^{2}\right\rangle=0.39$ and $\left\langle\xi_{a_{0}}^{4}\right\rangle=0.28$ within the threshold range. After $n_{c}=\frac{1}{2} \mathrm{fm}^{-4}$ turning on both moments are still well convergent and decrease to lower values: $\left\langle\xi_{a_{0}}^{2}\right\rangle=0.20$, $\left\langle\xi_{a_{0}}^{4}\right\rangle=0.17$. Similar to the case of $\xi_{K_{0}^{*}}$, at $n_{c}=1 \mathrm{fm}^{-4}$ we find there is also flipping of the second and fourth moments- $\left\langle\xi_{a_{0}}^{2}\right\rangle=0.01$ and $\left\langle\xi_{a_{0}}^{4}\right\rangle=0.07$ - which shows breakdown of convergence of moment, at $n_{c}=2 \mathrm{fm}^{-4}$ we can get $\left\langle\xi_{a_{0}}^{2}\right\rangle=-0.37$ and $\left\langle\xi_{a_{0}}^{4}\right\rangle=-0.13$ which shows negative value but the convergence recovers. The moments and LCDAs of $a_{0}$ are plotted in Fig. 5 and right panel of Fig.6, respectively.

The instanton-involved twist-3 LCDAs calculated in this work present nontrivial properties, to some extent is unexpected. Although in principle non-positive-definite LCDAs are allowed, since here we lack of direct test of these LCDAs in exclusive processes we would like to give some tentative discussion on the possible ingredients which are not mentioned above and may have some impacts on our results.

\section{- multi-instantons}


For simplicity we adopt single instanton approximation in our calculation, in fact there might be multi-instanton contribution to the correlation function. But the results in Ref.[39] indicate that in singular gauge multi-instanton contribution to the pion correlator is coincident with that given by single-instanton approximation. While physical results should be gauge-independent thus we conjecture it might be reasonable to utilize single-instanton approximation in our calculation. On the other hand on can easily see it is very difficult to work out the instanton contribution in singular gauge for $n \neq 0$.

\section{- instanton density}

It is obvious that the instanton density is crucial to obtain convergent results. Low instanton

density is welcomed in our calculation. The density $\frac{1}{2} \mathrm{fm}^{-4}$ is widely used under single instanton approximation which give many reasonable results. In a way this indicates that low instanton density is consistent with single approximation. In other word it seems that high instanton density is questionable at single instanton approximation. Maybe it is the main reason that at high density the convergence is lost.

\section{- subleading Fock states}

From Eq.(2) one can see we use valence model to investigate twist-3 LCDAs. The nonleading Fock states also may contribute twist-3 component via the mixing with other wave functions.[30]. This correction can be added by using the renormalization group, so it is less relative to this work.

\section{CONCLUSIONS}

In the present work we have investigated the instanton effect by single instanton approximation on the twist-3 LCDAs of pion, $f_{0}(1370), K_{0}^{*}(1430)$ and $a_{0}(1450)$ from valence quark model within the framework of QCD moment sum rules. Results illustrate that the instantonfree twist-3 LCDAs are always positive-definite while the instanton-involved LCDAs show some nontrivial properties. We find that low instanton density is consistent with the method adopted in this work. Possible ingredients which might have impact on the results are briefly discussed. Nonetheless we hope these LCDAs may be helpful to some heavy flavored exclusive processes 
since we conjecture the instanton density may play some role of a tuning parameter in deriving experiment favored results.

\section{ACKNOWLEDGEMENTS}

This work is partly supported by NNSFC under Project No. 10775117 and the Fundamental Research Funs for the Central Universities.

\section{Appendix A: Vanishing of the pure zero-mode contribution to tensor moment sum} rules

We still work in four-dimension Euclidean space. Considering the following two-pint correlation function

$$
\begin{aligned}
\int d^{4} x e^{i q x}\left\langle 0\left|T O_{n}(x) O^{\dagger}(0)\right| 0\right\rangle= & \frac{1}{256 \pi^{4} m_{1}^{*} m_{2}^{*}} \int d^{4} x e^{i q x} \int d \rho n(\rho) \rho^{4} \int d^{4} x_{0} \frac{1}{\left(x_{0}^{2}+\rho^{2}\right)^{3}} \\
& \times \frac{1}{\left[\left(x-x_{0}\right)^{2}+\rho^{2}\right]^{3 / 2}}\left(i z \cdot \overleftrightarrow{D}_{I}\right)^{n+1} \frac{1}{\left[\left(x-x_{0}\right)^{2}+\rho^{2}\right]^{3 / 2}} \\
& \times \operatorname{tr}\left[\gamma_{\mu} \gamma_{\nu}\left(1-\gamma_{5}\right) \sigma_{\alpha \beta} \gamma_{\sigma} \gamma_{\rho}\left(1-\gamma_{5}\right)\right] \\
& \times \operatorname{tr}\left[\tau_{\mu}^{+} \tau_{\nu}^{-} \tau_{\sigma}^{+} \tau_{\rho}^{-}\right]
\end{aligned}
$$

with

$$
O_{n}(x)=\bar{q}_{10}(x) \sigma_{\alpha \beta}\left(i z \cdot \overleftrightarrow{D}_{I}\right)^{n+1} q_{20}(x), \quad O^{\dagger}(0)=\bar{q}_{20}(0) q_{10}
$$

where the integrations over instanton collective coordinates are explicitly. In fact it is enough to concentrate on the trace part in Eq. (A1)

$$
\begin{aligned}
\operatorname{tr}\left[\tau_{\mu}^{+} \tau_{\nu}^{-} \tau_{\sigma}^{+} \tau_{\rho}^{-}\right]= & \operatorname{tr}\left[\left(\delta_{\mu \nu}+i \eta_{a \mu \nu} \tau^{a}\right)\left(\delta_{\sigma \rho}+i \eta_{b \sigma \rho} \tau^{b}\right)\right] \\
& =2 \delta_{\mu \nu} \delta_{\sigma \rho}-2 \eta_{a \mu \nu} \eta_{a \sigma \rho} \\
& =2\left(\delta_{\mu \nu} \delta_{\sigma \rho}-\delta_{\mu \sigma} \delta_{\nu \rho}+\delta_{\mu \rho} \delta_{\nu \sigma}\right)-2 \varepsilon_{\mu \nu \sigma \rho}
\end{aligned}
$$

where the use of Eq. (23) and Eq. (24) have been made. Then combining above result with the trace over $\gamma$ matrix we have

$$
\begin{array}{r}
{\left[2\left(\delta_{\mu \nu} \delta_{\sigma \rho}-\delta_{\mu \sigma} \delta_{\nu \rho}+\delta_{\mu \rho} \delta_{\nu \sigma}\right)\right.} \\
\left.-2 \varepsilon_{\mu \nu \sigma \rho}\right] \times 2 \operatorname{tr}\left[\gamma_{\mu} \gamma_{\nu}\left(1-\gamma_{5}\right) \sigma_{\alpha \beta} \gamma_{\sigma} \gamma_{\rho}\right] \\
=-2 \varepsilon_{\mu \nu \sigma \rho} \times 2 \operatorname{tr}\left[\gamma_{\mu} \gamma_{\nu}\left(1-\gamma_{5}\right) \sigma_{\alpha \beta} \gamma_{\sigma} \gamma_{\rho}\right]
\end{array}
$$


while in four-dimension Euclidean space

$$
\gamma_{5}=\frac{1}{4 !} \varepsilon_{\mu \nu \sigma \rho} \gamma_{\mu} \gamma_{\nu} \gamma_{\sigma} \gamma_{\rho}
$$

Now one can see the whole trace part in Eq. (A1) vanishes thus there is no pure zero-mode contribution to tensor moment sum rules consequently the LCDA.

[1] G. P. Lepage, S. J. Brodsky, Phys. Rev. Lett. 43, 545(1979); 43, 4625(E)(1979); Phys. Lett. B87, 359(1979), Phys. Rev. D 22, 2157(1980); S. J. Brodsky and G. Lepage, "Exclusive processes in quantum chromodynamics" in Perturbative quantum chromodynamics, edited by A. H. Mueller (World Scientific, Singapore, 1989).

[2] A. V. Efremov, A. V. Radyushkin, Phys. Lett, 94B, 245(1980).

[3] V. M. Braun and I. B. Filyanov, Z. Phys. C, 48, 239(1990).

[4] M. Beneke, G. Buchalla, M. Neubert and C. T. Sachrajda, Phys. Rev. Lett. 83, 1914(1999); Nucl. Phys. B591, 313(2000); Nucl. Phys. B606, 245(2001); M. Beneke, M. Neubert, Nucl. Phys. B675, 333(2003).

[5] H-Y. Cheng and K-C. Yang, Phys. Rev. D 71, 054020(2005);

[6] H-Y. Cheng, C-K. Chua and K-C. Yang, Phys. Rev. D 73, 014017(2006);

[7] H-Y. Cheng, C-K. Chua and K-C. Yang, Phys. Rev. D 77, 014034 (2008).

[8] M. A. Shifman, A. I. Vainshtein and V. I. Zakharov, Nucl. Phys. B147, 385(1979), B147, 448(1979).

[9] L. J. Reinders, H. Rubinstein and S. Yazaki, Phys. Rept. 127, 1(1985); S. Narison, QCD Spectral Sum Rules(World Scientifi, Singapore, 1989).

[10] A. A. Belavin, A. M. Polyakov, A. S. Schwartz and Yu. S. Tyupkin, Phys. Lett. 59B, 85(1975);

[11] G. 't Hooft, Phys. Rev. Lett. 37, 8(1976);

[12] G. 't Hooft, Phys. Rev. D 14, 3432(1976); 18, 2199(E)(1976);

[13] C. G. Callan, R. F. Dashen and D. J. Gross, Phys. Lett. 63B, 334(1976); Phys. Rev. D 17, 2717(1978).

[14] D. I. Dyakonov and V. Yu. Petrov, Nucl. Phys. B427, 457(1986).

[15] E. V. Shuryak, Nucl. Phys. B214, 237(1983). 
[16] T. Schäfer, E. V. Shuryak, Rev. Mod. Phys. 70, 323(1998), and the references therein.

[17] T. Schäfer, E. V. Shuryak, Phys. Rev. Lett. 75, 1707 (1995); H. Forkel and M. Nielsen, Phys. Lett. B345, 55(1995); L. Kinsslinger and M. B. Johnson, Phys. Lett. B523, 127(2001); F. Shi, T. G. Steele, V. Elias, K. B. Sprague et al, Nucl. Phys. A671 416(2000); G. Orlandini, T.G. Steele, D. Harnett, Nucl. Phys. A686 261(2001).

[18] H. Forkel, Phys. Rev. D 71, 054008(2005).

[19] J. Zhang, H. Y. Jin, Z. F. Zhang, T. G. Steele et al, Phys. Rev. D 79, 114033(2009).

[20] V. L. Chernyak and A. R. Zhitnitsky, Nucl. Phys. B201, 492(1982).

[21] V. L. Chernyak and A. R. Zhitnitsky, Phys. Rept. 112, 173(1984).

[22] V. M. Braun et al(QCDSF/UKQCD Collaboration), Phys. Rev. D 74, 074501(2006).

[23] B. Aubert et al. (BABAR Collaboration), Phys. Rev. D 70, 111102(2004); Phys. Rev. Lett. 94, 041802(2005); hep-ph/0408073; Phys. Rev. D 72, 052002(2005).

[24] A. Garmash at al.(Belle Collaboration), Phys. Rev. D 71, 092003(2005); A. Bonder(Belle Collaboration), hep-ph/0411004 K. Abe at al.(Belle Collaboration), hep-ph/0509003.

[25] Y. Kwon, G. Punzi and J. G. Smith(Particle Data Group) “ Production and Decay of b-Flavored Hadrons" in Ref. [37]; H-Y Cheng and J. Smith, Annu. Rev. Nucl. Part. Sci. 59, 215(2009).

[26] T. Schäfer and E. V. Shuryak, Phys. Rev. D 50, 478(1994); V. Yu. Petrov at al, Phys. Rev. D 59, 114018(1999); A. E. Dorokhov, JETP Lett. 77, 63(2003); S. Nam, H-C. Kim, A. Hosaka and M. M. Musakhanov, Phys. Rev. D 74, 014019(2006); S. Nam and H-C. Kim, Phys. Rev. D 74, 096007(2006);

[27] S. S. Agaev and M. A. G. Nobary, Phys. Rev. D 77, 074014(2008).

[28] S. V. Mikhailov, A. V. Pimikov and N. G. Stefanis, arXiv: peh-ph/1006.2936.

[29] T. Huang and X.G. Wu, Phys.Rev.D70, 093013(2004);T. Huang, M.Z. Zhou, X.H. Wu, Eur.Phys.J.C42, 271(2005); T. Huang, X.H. Wu, M.Z. Zhou, Phys.Rev.D70, 014013(2004);A. V. Radyushkin, Phys. Rev. D 80, 094009(2009);

[30] P. Ball, V. M. Braun and A. Lenz, JHEP. 05(2006)004;

[31] C-D. Lü, Y-M. Wang and H. Zou, Phys. Rev. D 75, 056001(2007).

[32] I. S. Gradshteyn, I. M. Ryzhik, Table of Integrals, Series and Products (Academic Press, Singapore, 2007), 7th edition. 
[33] P. Faccioli and E. V. Shuryak, Phys. Rev. D 64, 114020(2001).

[34] E. V. Shuryak, The QCD Vacuum, Hadrons and the Superdense Matter (World Scientific, Singapore, 2004), 2nd edition.

[35] M. Abramowitz and I. A. Stegun, Handbook of Mathematical Functions: with Formulas, Graphs and Mathematical Tables (Dover Publications, New York, 1972).

[36] A. Khodjamirian T. Mannel and M. Melcher, Phys. Rev. D 70, 094002(2004).

[37] Particle Data Group. C. Amsler et al, Phys. Lett. B667, 1(2008).

[38] H. Forkel and M. Nielsen, Phys. Lett. B345, 55(1995)

[39] A. E. Dorokhov, S. V. Esaibegyan, N. I. Kochelev N. G. Stefanis, J. Phys. G. 23, 643, (1997).

[40] M.-C. Chu, J. M. Grandy, S. Huang and J. W. Negele, Phys. Rev. D 49, 6039 (1994).

[41] M. Cristoforetti, P. Faccioli, M. C. Traini, J. W. Negele. Phys. Rev. D 75, 034008 (2007).

[42] V. M. Belyaev, and M. B. Johnson, Phys. Rev. D 56, 1481(1997).

[43] A. Mitov, Phys. Rev. D 71, 054021(2005).

[44] A. E. Dorokhov, W. Broniowski, and E. R. Arriola, Phys. Rev. D 74, 054023(2006).

[45] P. Colangelo, F. De Fazio and W. Wang, Phys. Rev. D 81, 074001(2010).

[46] K.- C. Yang, Nucl. Phys. B776, 187(2007).

[47] P. Ball1 and R. Zwicky, Phys. Rev. D 71, 014029(2005).

[48] A. Khodjamirian and R. Rckl, in Heavy Flavours, edited by A. J. Buras and M. Lindner (World Scientific, Singapore, 1998), 2nd edition.

[49] P. Faccioli, A. Schwenk and E. V. Shuryak, Phys. Lett, B549, 93(2002).

[50] P. Faccioli, A. Schwenk and E. V. Shuryak, Phys. Rev. D 67, 113009(2003).

[51] P. Faccioli, Phys. Rev. C 69, 065211(2004).

[52] L. S. Brown, R. D. Carlitz, D. B. Craemer and C Lee, Phys. Rev. D 17, 1583(1978). 